\title{
Synaptic vesicles studied by dynamic light scattering
}

\author{
S. Castorph ${ }^{1, a, b}$, S. Schwarz Henriques ${ }^{1, b}$, M. Holt ${ }^{2}$, D. Riedel ${ }^{3}$, R. Jahn ${ }^{2}$, and T. Salditt ${ }^{1, c}$ \\ 1 Institut für Röntgenphysik, Georg-August-Universität Göttingen, Göttingen, Germany \\ 2 Max Planck Institut für biophysikalische Chemie, Department of Neurobiology, Göttingen, Germany \\ 3 Max Planck Institut für biophysikalische Chemie, Electron Microscopy Group, Göttingen, Germany
}

Received 28 October 2010

Published online: 27 June 2011

(C) The Author(s) 2011. This article is published with open access at Springerlink.com

\begin{abstract}
The size polydispersity distribution of synaptic vesicles (SVs) is characterized under quasiphysiological conditions by dynamic light scattering (DLS). Highly purified fractions of SVs obtained from rat brain still contain a small amount of larger contaminant structures, which can be quantified by DLS and further reduced by asymmetric-flow field-flow (AFFF) fractionation. The intensity autocorrelation functions $g_{2}(\tau)$ recorded from these samples are analyzed by a constrained regularization method as well as by an alternative direct modeling approach. The results are in quantitative agreement with the polydispersity obtained from cryogenic electron microscopy of vitrified SVs. Next, different vesicle fusion assays based on samples composed of SVs and small unilamellar proteoliposomes with the fusion proteins syntaxin 1 and SNAP-25A are characterized by DLS. The size increase of the proteoliposomes due to SNARE-dependent fusion with SVs is quantified by DLS under quasi-physiological conditions.
\end{abstract}

\section{Introduction}

Synaptic vesicles (SVs) are small membranous organelles within the nerve terminal, encapsulating neurotransmitters by a lipid bilayer, and enabling a highly controlled fusion event with the synaptic membrane. The transport of the neurotransmitter, the fusion at the synaptic membrane, and the release of the stored neurotransmitters into the synaptic cleft are since long known as an essential step in nerve conduction of the chemical synapse [1-3]. A detailed structural view of these molecular mechanisms is still lacking, notwithstanding the enormous progress in the field during recent years [4-7]. For example, it is now known that proteins cluster and form micro-domains on the SV membrane. Among the protein inventory on the $\mathrm{SVs}$ is the fusiogenic R-SNARE protein synaptobrevin, forming complexes which are believed to control the fusion of the vesicles with the plasma membrane during exocytosis $[8,9]$, a highly spatially and temporally coordinated process. Membrane trafficking in eucaryotic cells follows common principles involving budding from a donor compartment and fusion with an acceptor compartment. Thus, the $\mathrm{SV}$ is regarded as a model trafficking organelle.

\footnotetext{
a e-mail: scastor@gwdg.de

b These authors contributed equally to this work.

c Present address: Institut für Röntgenphysik, Georg-August-Universität Göttingen, Friedrich-Hund-Platz 1, Göttingen, D-37077, Germany. e-mail: tsaldit@gwdg.de
}

The SV is one of the few organelles that can be purified to apparent homogeneity, and can be obtained in relatively large quantities employing well-established protocols [10] to isolate SVs from the presynaptic volume. The small size of the SVs with an average radius $R \simeq 21 \mathrm{~nm}$ [5], and their physical parameters enable size-fractionation techniques to work, incompatible with many other organelles. Moreover, the isolation procedure is relatively mild with no detergents involved. Importantly, SVs have been shown to be still functional after isolation, by neurotransmitter uptake and fusion assays.

Based on the opportunity to isolate SVs, quantitative measurements of size, content, mass distribution and protein composition of these highly compact multicomponent organelles have become possible. The lowresolution structure and size distribution of SV populations have been characterized by means of cryogenic electron microscopy (cryo-EM) [5] and synchrotron-based small-angle X-ray scattering (SAXS) data recorded from SV dispersions under quasi-physiological conditions [6].

SAXS can be performed with time resolution (in the order of a few ms) at the state-of-the-art synchrotron radiation sources. Since both size distribution and form factor are convoluted, usually the size distribution needs to be known in order to deduce structural details from SAXS spectra of polydisperse samples. However, the cryoEM analysis is invasive, costly and time consuming and does not allow real-time-resolved analysis of the SV size distribution. 
Dynamic light scattering (DLS) can be employed for detailed characterizations of vesicles and liposomes and has been extensively used as such to study vesicle sizes $[11,12]$ and size distributions $[13,14]$. Further, it is capable to detect and quantifiy the size increase after vesicle aggregation and fusion [15-18]. The scattering technique allows to obtain unbiased information averaged over a fairly large number of particles as compared to singleparticle imaging techniques like cryo-EM in reasonably short times (in the order of $100 \mathrm{~s}$ ), enabling to investigate large numbers of samples in a batch and promising to open up the possibility to follow the dynamics of polydispersity distributions in real time. The needed sample volumes for DLS are relatively small (about $1 \mu \mathrm{g}$ of SVs in about $1 \mathrm{ml}$ buffer). No invasive sample preparation steps are needed and DLS measurements are compatible with quasi-physiological conditions. Further, samples are reusable after measurements.

Here, we analyze DLS autocorrelation functions obtained from SVs under quasi-physiological conditions by three different methods: a single exponential fit assuming monodisperse particles; a regularized inversion (CONTIN) and a parameterized model based on EM data. We discuss the results and limitations of the three methods of analysis and compare our findings with previously reported SV polydispersities [14] and EM data. Thus, we find a second trace population of larger particles making up $45 \%$ or $79 \%$ of the total mass depending on the method of analysis. Asymmetric-flow field-flow (AFFF) fractionation was used here for further purification of the SV samples, reducing significantly the mass contribution of larger contaminant particles by a factor of 4 . The regularized inversion of DLS spectra of SV populations purified using this method yielded a size distribution which is in excellent agreement with cryo-EM and SAXS data. While the inversion approach worked well in the case of SV samples purified by means of AFFF, it failed to resolve the bimodal size distribution seen with the parameterized model in the case where larger trace particles are present in higher numbers in the sample. Instead, a mono-modal distribution was obtained, shifted slightly toward larger radii and showing a somewhat larger width.

Having assessed the performance of the regularized inversion, we apply it to the study of SV fusion, as it makes the least assumptions on the polydispersity of the sample. Previous studies have shown that the aggregation and fusion of SVs is detectable by DLS $[14,18]$. Here, we focus on small proteoliposomes which are fused in a SNAREdependent manner with SVs forming a cell free model fusion system [19]. From the measured size increase we calculate an average number of fusion events per proteoliposome and the fraction of fused SVs.

\section{Sample purification and preparation}

\subsection{Synaptic vesicle purification}

Synaptic vesicles from rat brain were purified following a purification protocol by Takamori et al. [5]. An addi- tional final centrifugation step was introduced to allow for buffer exchange. To this end, the centrifuged pellets were re-suspended in HB100 buffer $(100 \mathrm{mM} \mathrm{KCl}, 25 \mathrm{mM}$

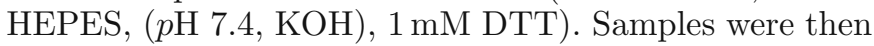
kept on water/ice mixtures until investigation by cryo-EM and DLS.

A constant (10:5:2) ratio of proteins, phospholipids and cholesterol is assumed for all SVs, according to [5]. The particle concentration of the SV populations was obtained by measuring the protein mass by a modified Lowry assay [20], plus the calculated contribution from membrane lipids and cholesterol. The resulting SV stock solutions had a protein concentration in the range of 3.68 to $4.56 \mu \mathrm{g}$ per $\mu \mathrm{l}$, corresponding to SV particle concentrations of about $2.09 \times 10^{14}$ to $2.66 \times 10^{14} \mathrm{SVs} / \mathrm{ml}$.

No aggregation was observed by EM (data not shown). However, a small number of larger membranous particles was observed, which seemed to be unavoidable in the purification protocol, e.g. due to limitations in the resolution of size exclusion chromatography. These particles may be formed by larger membrane aggregates, possibly originating from early endosomes. For further details see $[5,6]$.

\subsection{Asymmetric-flow field-flow fractionation}

Asymmetric-flow field-flow (AFFF) fractionation is a onephase chromatography technique allowing to separate particles of different sizes on the basis of their diffusion properties [21]. AFFF has been used for characterization and size fractionation of vesicles [22-26], including applications to biological vesicles $[27,28]$. The dispersed particles travel along a channel with a parabolic velocity profile in the principal flow direction. An additional perpendicular force field is applied, which drives the particles toward one wall of the flow channel. The particles exhibit an equilibrium position in the direction of the perpendicular force field which depends on their diffusion associated with Brownian motion. Thus, the particles exhibit different travel velocities in the direction of the principal flow. Smaller particles will reach the end of the channel faster than larger particles, and subsequent sample fractions containing particles of different sizes can be collected. Importantly, the sample does not interact with a stationary phase which might alter the sample. AFFF is a robust and quick technique, needing only low-sample amounts. Further, the sample is reusable in other experiments.

An Eclipse 2 system from Wyatt Technology is used for the AFFF fractionation with a channel of height $350 \mu \mathrm{m}$ and length $275 \mathrm{~mm}$. A precut membrane of regenerated Cellulose ( $10 \mathrm{kDa}$ molecular weight cut-off) is used. The Eclipse system is connected to an Agilant 1100 series HPLC pump, and to a DAWN EOS multi-angle light scattering setup. The channel flow is constantly $1.00 \mathrm{ml} / \mathrm{min}$. The sample is injected with an inject flow rate of $0.20 \mathrm{ml} / \mathrm{min}$ for $1 \mathrm{~min}$, and focused with a focus flow rate of $3 \mathrm{ml} / \mathrm{min}$ for $2 \mathrm{~min}$. Subsequently, the cross flow is set to $0.5 \mathrm{ml} / \mathrm{min}$ and is linearly decreased to $0 \mathrm{ml} / \mathrm{min}$ over $40 \mathrm{~min}$. Subsequent fractions are collected for $60 \mathrm{~s}$ each, starting $3 \mathrm{~min}$ after the injection of the SV 
sample, and $8 \mathrm{~min}$ after the initiation of the fractionation procedure. The total duration of one fractionation procedure was $40 \mathrm{~min}$.

For the AFFF fractionation, SV stock solution is diluted in AFFF buffer $(150 \mathrm{mM} \mathrm{KCl,} 20 \mathrm{mM} \mathrm{HEPES}$, $\left.(p \mathrm{H} 7.4, \mathrm{KOH}), 0.02 \% \mathrm{NaN}_{3}\right)$, giving a total final protein concentration of $0.72 \mathrm{mg} / \mathrm{ml}$. About $22 \mu \mathrm{g}$ of protein are used for each AFFF run.

\subsection{Preparation of proteoliposomes}

Lipids were obtained from Avanti Polar Lipids and Molecular Probes and were mixed in chloroform giving molar ratios (given in brackets) resembling that of native SVs according to [5], i.e. phosphatidyline (5), phosphatidylethanolamine (2), phosphatidylserine (1), phosphatidylinositol (1). Unlabeled phosphatidylethanolamine (PE) was partly substituted by N-(7-nitro-2,1,3-benzoxadiazol4-yl) PE (NBD-PE) and Rhodamine-PE [29]. After drying, lipids were resuspended in HB100 buffer with $5 \%$ sodium cholate at a total lipid concentration of $13.5 \mathrm{mM}$. SNARE proteins (Syntaxin 1 and SNAP-25A) were cloned with rat (Rattus norvegicus) sequences as templates and expressed in E. coli, and subsequently purified [29]. SNARE proteins were added at a physiologically relevant SNARE:phospholipid molar ratio of 1:300 [5] to the cholate micellar solution. Detergent was removed by gel filtration chromatography on a SMART system with a PC 3.2/10 Fast Desalting column (GE Healthcare). For further details on the proteoliposome preparation, see [29] and associated on-line supplemental data.

\section{Experiments}

\subsection{Instrumentation and choice of parameters}

If not indicated differently, the DLS measurements were performed at SVs dispersed in HB100 buffer. For the DLS measurements of the SV sample used for the AFFF fractionation, a SV stock solution of $3.58 \mu \mathrm{g} / \mu \mathrm{l}$ is diluted by a factor of 1000 with degassed aqueous AFFF buffer, leading to a total protein concentration of $3.58 \mu \mathrm{g} / \mathrm{ml}$. Collected AFFF fractions are not further diluted for DLS measurements since the fractionation process already leads to a dilution factor of about 1000. The DLS measurement results are relatively insensitive to the exact particle concentration of SVs within a relatively large range, see appendix B.

For the fusion experiments, a SV stock solution of $4.56 \mathrm{mg} / \mathrm{ml}$ protein concentration is diluted with degassed aqueous HB100 buffer (see above) to a total protein concentration of $0.83 \mu \mathrm{g} / \mathrm{ml}$. Note that the SV samples for the fusion experiments were not purified by means of AFFF fractionation. For the fusion reactions, Syntaxin 1/SNAP25 proteoliposomes (NBD-Rhodamine labeled) are added to the SV solutions at about 0.4 times the total mass concentration as the SVs.
Fusion activity was checked in a similar manner like in [29] by fluorescence dequenching assays, and the size increase of the fused SV/liposomes was determined by cryoEM (data not shown). Vesicle clusters have been absent in electron micrographs of similar samples [29], indicating that aggegation of vesicles does not occur in significant amounts. For the inhibited fusion reactions, SVs are incubated for $60 \mathrm{~min}$ at $37^{\circ} \mathrm{C}$ in $\mathrm{HB} 100$ with Tetanus toxin (TeNT), which targets Synaptobrevin. The enzyme was added in a molar ratio of 1:50 with regard to the concentration of Synaptobrevin [29]. Samples for the fusion reaction are incubated under similar conditions in HB100 (no addition of TeNT).

The samples are put into cylindrical borosilicate cuvettes with a diameter of $10 \mathrm{~mm}$ (Fisher Scientific), and are closed air tight with polymer caps (Carl Roth GmbH, Karlsruhe, Germany). DLS is performed with an ALV/CGS-3 Laser Light Scattering Goniometer System (ALV-GmbH, Langen, Germany), equipped with a $22 \mathrm{~mW}$ HeNe-Laser $(\lambda=632.8 \mathrm{~nm}$, UNIPHASE, model 1145P), and an ALV-7004 or ALV-5004 Multiple Tau Digital Correlator. The scattered intensity is recorded by a high quantum efficiency avalanche photo diode at a scattering angle of $90^{\circ}$ in the scattering plane, perpendicular to the vertically polarized incident beam. If not indicated differently, the intensity autocorrelation function calculated for three consecutive intervals of $30 \mathrm{~s}$ is averaged, corresponding to $90 \mathrm{~s}$ accumulation time. Three such runs are then performed to obtain the final averaged intensity autocorrelation function $g_{2}(\tau)$, representing a total measurement time of $270 \mathrm{~s}$. The errors are calculated by the standard deviation $\sigma_{\tau}$ of the nine runs for each $\tau$. The resulting (normalized amplitude) correlation function $g_{1}(\tau)$ is given by $\beta\left|g_{1}(\tau)\right|=\sqrt{g_{2}(\tau)-1}[30,31]$ with the intensity correlation function $g_{2}(\tau)=\langle I(t) I(t+\tau)\rangle_{t} /\langle I\rangle_{t}^{2}$ and the coherence factor $\beta$.

\subsection{Data analysis}

For a dilute suspension of polydisperse spherical particles of hydrodynamic radii $R_{h}$ in Brownian motion, $g_{1}(\tau)$ is the Laplace transform with respect to the hydrodynamic radii $R_{h}$ given by [31]

$$
g_{1}(\tau)=\int \mathrm{d} R_{h} p_{I}\left(R_{h}\right) \exp \left(\frac{-k_{B} T}{6 \pi \eta R_{h}} q^{2} \tau\right),
$$

with $p_{I}\left(R_{h}\right)$ the intensity weighted distribution of diffusion constants, $k_{B}$ Boltzmann's constant, $T$ temperature, and $\eta$ the viscosity of the buffer. Considering that the inverse Laplace transformation of $g_{1}(\tau)$ with respect to time is a well-known "ill-conditioned" problem [32], we choose different approaches to tackle this problem and to analyze $g_{2}(\tau)-1$. Table 1 gives an overview of the different models optimized against $g_{2}(\tau)-1$. See appendix A for details on the models and the data analysis. 
Table 1. Overview about different models tested in the analysis of DLS data from synaptic vesicles. See appendix A for further details on the models and the data analysis.

\begin{tabular}{|c|c|c|c|c|}
\hline $\begin{array}{l}\text { Analysis } \\
\text { model }\end{array}$ & $\begin{array}{l}\text { Synaptic Vesicle } \\
\text { model }\end{array}$ & $\begin{array}{l}\text { Size polydispersity } \\
\text { model }\end{array}$ & Advantages & Disadvantages \\
\hline $\begin{array}{l}\text { Single } \\
\text { exponential }^{\mathrm{a}}\end{array}$ & $\begin{array}{l}\text { Spherical } \\
\text { particle }\end{array}$ & Monodisperse & $\begin{array}{l}\text { Accurate for } \\
\text { monodisperse } \\
\text { samples }\end{array}$ & $\begin{array}{l}\text { Bias towards larger } \\
\text { radii in polydisperse } \\
\text { samples }\end{array}$ \\
\hline $\begin{array}{l}\text { Regularized } \\
\text { inversion }\end{array}$ & $\begin{array}{l}\text { Solid or } \\
\text { hollow } \\
\text { sphere }^{\text {b }}\end{array}$ & $\begin{array}{l}\text { Model free, } \\
\text { but regularized } \\
\text { (simplest solution) }\end{array}$ & $\begin{array}{l}\text { No assumptions on } \\
\text { polydispersity of } \\
\text { sample }\end{array}$ & $\begin{array}{l}\text { Shape of size distribution } \\
\text { influenced by regularization; } \\
\text { artifact peaks at roots of } \\
\text { particle form factor }\end{array}$ \\
\hline $\begin{array}{l}\text { Parameterized } \\
\text { models }\end{array}$ & $\begin{array}{l}\text { Hollow } \\
\text { sphere }^{\text {b }}\end{array}$ & $\begin{array}{l}\text { Cryo-EM data } \\
+ \text { Gaussian }\end{array}$ & $\begin{array}{l}\text { Accurate control for/ } \\
\text { correction of } \\
\text { previously measured } \\
\text { size distributions }\end{array}$ & $\begin{array}{l}\text { Model for shape of } \\
\text { polydispersity must be } \\
\text { previously known }\end{array}$ \\
\hline
\end{tabular}

\footnotetext{
a See appendix A.

b Shell thickness $12 \mathrm{~nm}$.
}

\section{Results}

\subsection{Regularized inversion}

Figure 1(A) shows the size distribution functions of a native SV preparation (solid black line) and from a selection of AFFF fractions, collected every other minute over $60 \mathrm{~s}$. The first fraction shown was collected 5 min after injection of the SV sample (chronological order of fractions, with their time of collection (in minutes) after injection of the SV sample: red crosses $(5 \mathrm{~min})$, blue full squares (7 min), orange empty squares $(9 \mathrm{~min})$, magenta full triangles (11 min), purple full diamonds (13 min), light gray plus signs $(15 \mathrm{~min}))$.

The SV samples used for the AFFF fractionation and for the collection of the DLS data depicted in fig. 4 were taken from the same vesicle preparation, see appendix A. The size distribution functions were calculated by the ALV software and then rescaled to show the relative abundance of vesicles between fractions. For each fraction the scaling factor $s$ was computed with the time-averaged intensity $\langle I\rangle_{t}: s=\langle I\rangle_{t} / \sum_{R_{h}} p_{I}\left(R_{h}\right)$. In a final step, the distribution functions were normalized such that the peak of the most abundant fraction was set to 1 . For comparison the size distribution of the unfractionated sample was included into the plot (solid black line), which is in excellent agreement with previous results obtained by DLS from similar SV samples [14].

The bulk of the SVs in fig. 1(A) appears in the first fractions (red crosses $(5 \mathrm{~min})$, blue full squares $(7 \mathrm{~min})$ ). The difference between the peak position of the two size distribution functions obtained from fractions ( $5 \mathrm{~min}$ ) and $(7 \mathrm{~min})$ is within the accuracy of the regularized fit. These fractions contain purified vesicles, the number of larger particles $(>60 \mathrm{~nm})$ is significantly reduced when compared to the number of larger particles in the unfractionated sample, indicating the successful fractionation of SVs away from larger particles. Mass distributions calculated from the regularized fit (see appendix A) suggest that the larger particles present to $45 \%$ in the native SVs before fractionation are reduced in the first fractions ( $5 \mathrm{~min}$ ) after fractionation to levels below the resolution of the regularized fit. Later fractions contain only fewer SVs and mostly larger particles.

Figure 1(B) shows the AFFF fraction ( 5 min), collected in minute 5 after injection of the SV sample, measured by DLS (red crosses) and the size distribution of SVs in unfractionated samples determined by cryo-EM (gray circles) along with a repetition from fig. 4 of the size distribution function of the unfractionated sample measured by DLS (solid black line). The cryo-EM data on the radius of the SVs was corrected for the expected effects of the outer proteins on the hydrodynamic radius $R_{h}$ by adding $3 \mathrm{~nm}$.

The size distribution as determined by cryo-EM agrees well with the AFFF fraction ( $5 \mathrm{~min}$ ). The latter extends somewhat toward larger particles. The distribution function of the unfractionated sample exhibits stronger deviation from the cryo-EM data and the single AFFF fraction data. In particular, there is a significantly higher number of larger particles present when compared to the number of larger particles present in the size distribution function obtained for the particles in AFFF fraction ( 5 min).

\subsection{Parameterized models}

Figure 2(A) shows the correlation functions $g_{2}(\tau)-1$ for a typical SV sample (green curve) and for a typical individual AFFF fraction obtained from the native SV sample 
(A)
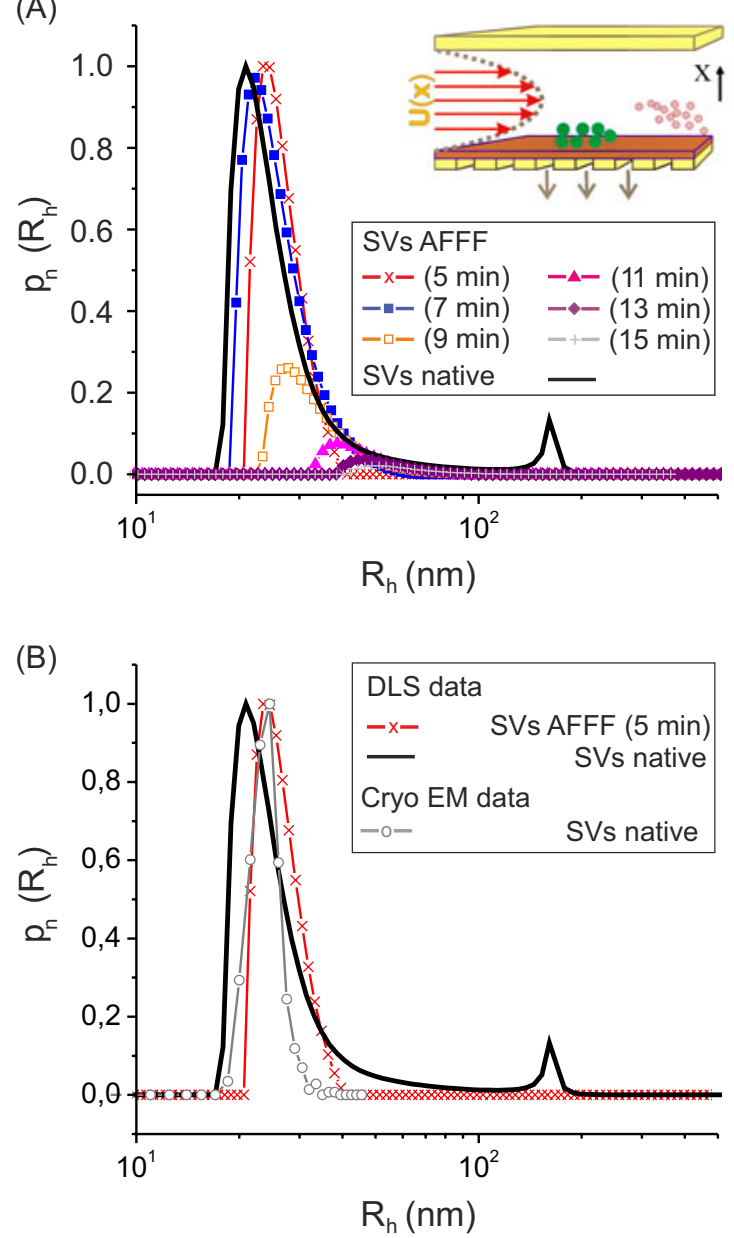

Fig. 1. (Colour on-line) (A) Size distribution of SVs by DLS. Native SV preparation (solid black line) and individual AFFF fractions (colored lines, time of collection (in minutes) after injection of the SV samples: red crosses (5 min), blue full squares ( $7 \mathrm{~min})$, orange empty squares $(9 \mathrm{~min})$, magenta full triangles (11 min), purple full diamonds (13 min), light gray plus signs $(15 \mathrm{~min})$ ) weighted by the relative number of particles within the corresponding individual AFFF fraction as determined from the time-averaged scattering intensity $\langle I\rangle_{t}$. The inset shows a schematic of the AFFF flow channel. Red arrows indicate the velocity $U(x)$ of the buffer in the direction of the flow channel, brown arrows indicate the direction of the channel cross-flow. (B) Size distribution of native SV preparation by DLS (solid black line) and cryo-EM (gray circles), shifted as detailed in the text, and individual AFFF fraction ( 5 min) from the shown native SV preparation (red crosses).

(black curve), exhibiting the characteristic (exponential) decay expected for polydisperse colloidal particles undergoing Brownian motion, along with the errors estimated from the different runs and a least-square fit of a model as detailed below (solid red line and dashed red line). Figure 2(B) shows the resulting bimodal distribution function $p_{n}\left(R_{h}\right)$ of the SVs (solid blue line, cryo-EM data, fixed during fitting) and of the larger aggregated membrane particles (dashed red line and solid red line). The corresponing mass fractions of the larger particles in the
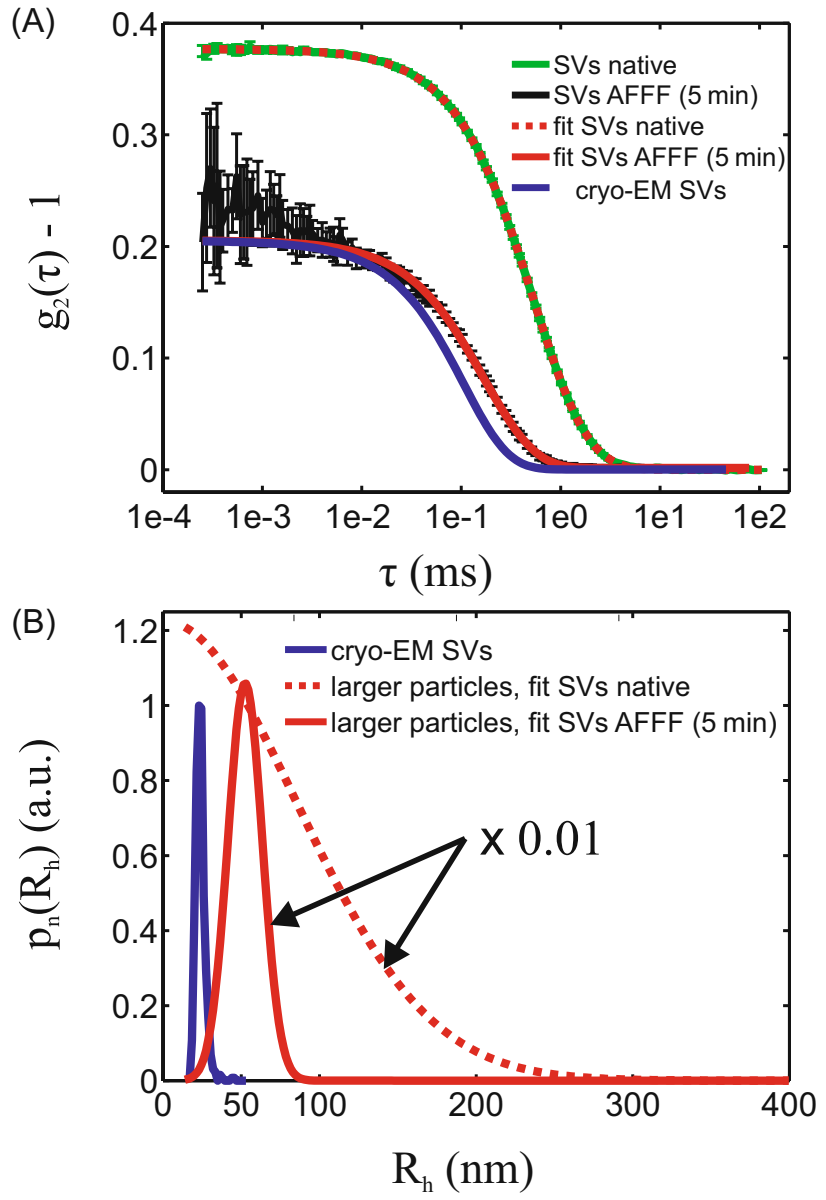

Fig. 2. (Colour on-line) (A) DLS data of native SV ensemble (green curve) and AFFF fraction (5 min) (black curve), collected in minute 5 after injection of the SV sample, with error bars and fitted model calculations (dashed red curve, solid red line). Calculated correlation function for particles following the cryo-EM size distribution (solid blue line). (B) Number-weighted bimodal size distribution functions consisting of the blue branch (cryo-EM data, smoothed) and one of the red branches (fitted Gaussian distributions, red solid line, dashed red curve) corresponding to the fits in (A), (red solid line, dashed red curve). Note the different scalings for the blue branch and the red branches.

samples are calculated to be about $79 \%$ and $23 \%$, respectively. The first value, which should be compared to the value of $45 \%$ obtained by the regularisation fit, shows that these numbers depend strongly on the choice of the model. The cryo-EM data is taken from [6]. Note the different scalings of the two components of $p_{n}\left(R_{h}\right)$, indicating that the large particles can be viewed as a small contamination when considering the number of particles.

The SVs as well as the larger membranous fragments were modeled as spherical core-shell particles with a shell thickness of $12 \mathrm{~nm}$, see appendix A for further details. This way the small unilamellar vesicle structure of the SVs and the membranous character of the larger particles is modeled. 


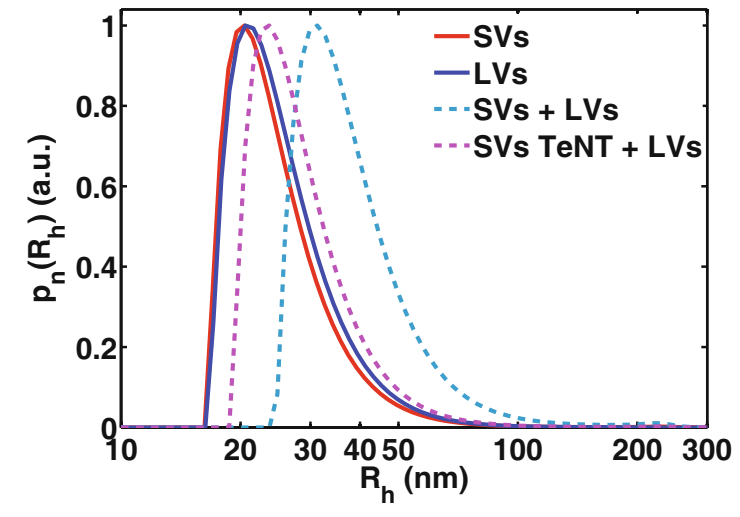

Fig. 3. (Colour on-line) DLS data of native SV ensemble (solid red line), LV preparation (solid blue line), mixture of SVs plus LVs (dashed cyan line) and TeNT-treated SVs plus LVs (dashed magenta line).

Using this model form factor a forward calculation approach was implemented to analyze the SV data, circumventing problems usually arising from the standard inversion approach of $g_{1}(\tau)$ implemented in most commercial DLS instruments. For the calculations, the SV size distribution obtained by cryo-EM (smoothed) was used, shifted by $3 \mathrm{~nm}$ toward lager radii to account for the size increase of the hydrodynamic radius resulting from proteins facing outwards of the SV, which were not taken into account in the EM size measurement.

The contribution from the larger membranous particles was parametrized with a Gaussian-shaped size distribution of particles with radii $R_{h} \geq 14 \mathrm{~nm}$. Together with the main population of isolated and intact SVs this second component formed a bimodal size distribution. Baselines for $\tau \rightarrow 0$ and $\tau \rightarrow \infty$ were fitted to $g_{2}(\tau)-1$. $p_{n}\left(R_{h}\right)$ was optimized by a least-squares fit to $g_{2}(\tau)-1$, solely by adjusting the position, width and relative height of the Gaussian size distribution of the larger particles to the $\mathrm{SV}$ population. The size distribution and relative contribution of the larger membranous particles depend to some extent on the individual SV preparation. The resulting calculated autocorrelation curves are found to be in excellent agreement with the measured $g_{2}(\tau)-1$ for the native SV ensemble (reduced $\chi^{2}=1.36$ ), and for the individual AFFF fraction (reduced $\chi^{2}=0.33$ ). In the latter case, the small value of $\chi^{2}$ may be due to somewhat overestimated measurement errors.

\subsection{Fusion experiments}

Figure 3 shows the size distribution functions $p_{n}\left(R_{h}\right)$ obtained by DLS from a polydisperse population of small unilamellar liposomes (LVs, solid blue line), native SVs (solid red line) as well as the product of a fusion reaction between LVs and SVs (dashed cyan line) and a corresponding control with TeNT-treated SVs added to LVs (dashed magenta line). The samples were not AFFF fractionated. The data has been analyzed using the ALV software, describing the particles as hard spheres.
Table 2. Model parameters as obtained from the fusion experiments by inversion of DLS data. Most frequent radius $R_{h}(\mathrm{~nm})$ of particles. The number of fusion events $\mathrm{N}$ (per LV) is calculated as $N=\left(R_{h, f}^{2}-R_{h, L V s}^{2}\right) / R_{h, S V s}^{2}$, where $R_{h, f}$ denotes the most frequent radius of the mixture of SVs and LVs, or of the mixture of NeNT treated SVs and LVs. Conservation of particle surface areas, and size homogeneity of SVs, LVs and fusion products is assumed, respectively. The fraction of fused SVs, $\mathrm{F}(\%)$, is calculated from $\mathrm{N}$ and the initial ratio of the numbers of SVs and LVs in the samples as determined by the protein and lipid concentrations taking into account the composition of the SVs [5] and LVs.

\begin{tabular}{lccc}
\hline Sample & $R_{h}(\mathrm{~nm})$ & $\mathrm{N}($ per LV) & $\mathrm{F}(\%)$ \\
\hline SVs & 21 & - & - \\
LVs & 21 & - & - \\
SVs + LVs & 31 & 1.2 & 60 \\
SVs TeNT treated + LVs & 24 & 0.3 & 15 \\
\hline
\end{tabular}

The size distribution functions $p_{n}\left(R_{h}\right)$ of all four samples are similar in shape and width. The most frequent radii of both the $\mathrm{LV}$ and $\mathrm{SV}$ samples are about $R_{h}=$ $21 \mathrm{~nm}$. The size distribution of the fusion products of $\mathrm{SVs}$ and LVs is shifted toward larger radii (maximum around $R_{h}=31 \mathrm{~nm}$ ) when compared to the size of the SV or LV ensembles. The size distribution function obtained from the sample containing TeNT-treated SVs and LVs is also slightly shifted toward larger radii (maximum around $R_{h}=24 \mathrm{~nm}$ ) when compared to the size of the $\mathrm{SV}$ or LV ensemble. However, the size shift toward larger radii relative to the $\mathrm{SV}$ or $\mathrm{LV}$ ensembles is significantly decreased for the sample containing TeNT-treated SVs and LVs when compared to the sample containing SVs and LVs. As expected, TeNT treatment of SVs inhibits effectively fusion between SVs and LVs. However, some residual fusion of TeNT-treated SVs with LVs might remain due to a small number of uncleaved SNAREs on the $\mathrm{SV}$, as one single SNARE complex per SV is sufficient for membrane fusion [33].

Table 2 gives the most frequent radii $R_{h}(\mathrm{~nm})$ and a characteristic number $\mathrm{N}$ (per LV) of fusion events of LVs and SVs, calculated from the shift of the most frequent radius when compared to the $\mathrm{LV}$ sample.

\section{Discussion and summary}

From the dilution series shown in appendix B, fig. 5(A), significant effects due to SV concentration on the DLS data within the investigated concentration range are excluded. Data obtained from the same SV sample are very reproducible, see fig. $5(\mathrm{~B})$.

A fit of a single exponential to intensity correlation data from SVs (appendix A, fig. 4(A)) gives a first estimate of the typical size range of the SVs. However, as expected, due to the oversimplistic model assumptions employed, the typical particle size obtained by the single ex- 
ponential fit systematically overestimates the actual mean particle size.

Regularized nonlinear inversion of the intensity correlation function by the ALV software (fig. 4(A) and (B)) gives access to the intensity-weighted size distribution function $p_{I}\left(R_{h}\right)$, or number-weighted size distribution function $p_{n}\left(R_{h}\right)$ of the $\mathrm{SV}$ population. Width and shape depend to some extent on the regularization. However, the maximum of $p_{n}\left(R_{h}\right)$ is hardly affected by different regularization parameters. It accords with EM data, which were also used for the direct modeling approach. Due to imperfections in the consideration of the form factor model, a second peak at a radius of about $160 \mathrm{~nm}$ is present in $p_{n}\left(R_{h}\right)$. This peak occurs in all cases, where $p_{I}\left(R_{h}\right) \neq 0$ for $F\left(q, R_{h}\right)=0$ and is more pronounced in cases where the particles are modeled as hollow hard spheres as compared to hard spheres. A precise assessment of the relative particle number of sizes within the range of the artificial peak is waived here. Results are found to be in excellent agreement with results from previous DLS studies of similar SV samples [14], which obtained a similar monomodal size distribution function accounting for contributions from both SVs and larger particles in the sample.

In a direct modeling approach, DLS spectra of SV dispersions can be consistently described with a bimodal size distribution (fig. 2(A) and (B)) based on cryo-EM data obtained at vitrified SV dispersions and a second Gaussiandistributed branch effectively modeling effects due to few larger trace particles of sizes in the order of up to a few $100 \mathrm{~nm}$ present in the sample. Note the inherent sensitivity of DLS for scattering contributions originating from larger particles due to the highly nonlinear dependence of the scattering intensity on the particle radius.

The mass fraction of larger contaminant particles in the SV samples with diffusion properties different from SVs are significantly reduced by AFFF from $79 \%$ to $23 \%$ according to the direct modeling approach and from $45 \%$ to levels below the resolution according to the regularized inversion. The reduction in mass by a factor of 4 greatly decreases the contribution from the tail toward larger radii in $p_{n}\left(R_{h}\right)$ (solid black line and red crosses in fig. 1(B)). Results are in excellent agreement with values reported previously [5].

Thus, the AFFF separation process is found to discriminate well between SV and larger trace particles. $\mathrm{AFFF}$ is found to be suited for further purification of SV samples and is showing great potential for enabling sizedependent analysis of SVs in the future.

However, the regularized size distribution obtained by DLS after AFFF purification extends still somewhat further toward larger radii (red crosses in fig. 1(B)), or a very small additional contribution of somewhat larger particles is needed (solid red line in fig. 2) when compared to the cryo-EM size data. This may be due to an underestimation of the number of larger SV particles by cryo-EM due to undersampling, and reflects the effects of very few remaining larger particles in the sample. In case of the inversion analysis, it cannot be excluded that part of the small deviation between the size distribution obtained by
cryo-EM (gray circles in fig. 1(B)) when compared to the one obtained by DLS (red crosses in fig. 1(B)) is also due to regularization effects.

The size increase after SV aggregation and fusion has previously been detected by DLS $[14,18]$. Here, we have used DLS to study the fusion of small proteoliposomes with SVs in a SNARE-dependent manner (fig. 3). From the measured size increase we calculated an average number of 1.2 fusion events per proteoliposome. Fusion was significantly reduced in a control experiment, where the SNAREs of SVs were cleaved with TeNT. Aggregation as a possible alternative explanation of the measured size increase was ruled out by the absence of vesicle clusters in electron micrographs of similar samples [29].

In summary, DLS spectra recorded from SVs under quasi-physiological conditions can be consistently described with a size distribution obtained by cryo-EM at vitrified SV dispersions. The effects originating from few larger trace particles of sizes in the order of few $100 \mathrm{~nm}$ in samples can be effectively modeled by a second Gaussiandistributed branch in the particle size distribution. The SV size distribution is rather sharp, compared to the rather broad size distribution of the larger membranous particles. Regularization and inversion of DLS spectra from samples containing few larger trace particles in turn lead to a mono-modal size distribution with a significantly overestimated width when compared to the SV size distribution obtained by cryo-EM. Although the position of the maximum of the size distribution is also slightly shifted toward larger radii, the result of regularized inversion is found to be still a fairly good estimate for the most likely radius within the SV population. Importantly, the size polydispersity distribution of the SVs by regularized inversion is obtained without assuming a particular shape of the size distribution function. Larger trace particles contaminating the SV population can be removed effectively by AFFF fractionation, giving access to diluted SV dispersions of very high purity. DLS spectra from AFFF fractions containing the SV population can be analyzed by means of regularization and inversion and reveal a SV size distribution consistent with cryo-EM data. With the polydispersity data of pure SVs and validated analysis at hand, one can then go ahead to investigate more complex samples, leading to cell-free fusion assays. To this end, we have investigated SNARE-dependent fusion of SVs with small unilamellar proteoliposomes by DLS. The fact that we could reliably detect the size increase associated with SNARE-dependent fusion confirms previous findings that DLS is very suitable for detecting aggregation and fusion in a whole range of cell-free assays $[14,18]$, potentially in real time. Importantly, in contrast to other more invasive techniques the samples can be recovered after the experiments and be used for biochemical analysis.

The authors thank Matias Hernandez for technical support regarding parameter settings and the handling of the AFFF apparatus. This work was financially supported by the Excellence Cluster Initiative 171/Deutsche Forschungsgemeinschaft (DFG) Research Center 103, Center for Molecular Physiology of the Brain, DFG SFB 803, and the Max Planck Society. 


\section{Appendix A. Data analysis}

Different models were calculated and optimized against $g_{2}(\tau)-1$. First, a single exponential decay $y(t)=y_{0}+$ $a e^{-t / \tau}$ is fitted to $g_{2}(\tau)-1$. From the fit result we obtain a single radius $R_{h}=2 q^{2} \tau k_{B} T /(6 \pi \eta)$. The fit region is chosen to lie within a range of $250 \mathrm{~ns}$ and $78.6 \mathrm{~ms}$ lag time.

Second, we calculate a nonlinear fit to $g_{2}(\tau)-1$ by using a constrained regularization method [34-36], employing a CONTIN algorithm $[37,38]$ in a standard implementation (ALV-Correlator Software ALV-7004 for Windows, V.3.0.4.5) by ALV GmbH, Langen, Germany. For the data analysis, several settings are specified within the $A L V$-Regularized fit setup of the ALV-Correlator Software. If not indicated differently, the fit model DLS-Exponential $\left(g_{2}(t)\right)$ is used, and fit additional baseline, zero-order regularizor, as well as enable data weighting is enabled. Further, the regularization strength is optimized by selecting single fit with target $P R O B 1=0.5$. Values from 0.1 to 0.9 for PROB1 have been tested and corresponding results have been compared. The fit range is chosen to be between $250 \mathrm{~ns}$ and $78.6 \mathrm{~ms}$. To estimate the errors of the correlation function at each lag time, the ALV software uses a theoretical model described in [32,39, 40].

The particles are modeled as spherical core-shell particles or as solid spheres. While the first particle model is a more realistic description of the synaptic vesicles the implementation of the latter displays less pronounced artificial peaks in the calculated size distributions arising from imperfections in the consideration of the form factor model. Both models yield similar results, a finding which can be explained based on the same $R^{6}$ dependence of the weight which a certain vesicle fraction $p_{I}(R) \mathrm{d} R$ has on the scattering intensity. In the core-shell model the shell thickness is set to $12 \mathrm{~nm}$, accounting for both lipid and protein components of the small unilamellar vesicle structures. The lipid bilayer thickness is taken to be about $6 \mathrm{~nm}[6]$, and the contributions from the protein shells are taken into account by an addional $3 \mathrm{~nm}$ on both sides of the bilayer.

Correspondingly, fit for vesicles with $r^{*}=12 \mathrm{~nm}$ was enabled, or disabled within the $A L V$-Regularized fit setup of the ALV-Correlator Software. The result of the inversion is the intensity-weighted distribution function $p_{I}\left(R_{h}\right)$, a measure of the contribution from the differently sized vesicles to the autocorrelation function. To obtain a size distribution function $p_{n}\left(R_{h}\right), p_{I}\left(R_{h}\right)$ needs to be corrected for the particle form factor amplitude $F\left(q, R_{h}\right)$ and volume $V\left(R_{h}\right)$ of the particle shell, or the particle (hard sphere),

$$
p_{n}\left(R_{h}\right)=\frac{p_{I}\left(R_{h}\right)}{V\left(R_{h}\right)^{2}\left|F\left(q, R_{h}\right)\right|^{2}} .
$$

As the roots in $F\left(q, R_{h}\right)$ would lead to singularities in $p_{n}\left(R_{h}\right)$, the particle form factor is smoothed around these points. Nevertheless artificial peaks can appear in $p_{n}\left(R_{h}\right)$ at the roots of $F\left(q, R_{h}\right)$. Both $p_{I}\left(R_{h}\right)$ and $p_{n}\left(R_{h}\right)$ are calculated by the ALV software. From $p_{n}\left(R_{h}\right)$ we calculated the mass distribution function $p_{M}\left(R_{h}\right)=p_{n}\left(R_{h}\right)$. $m\left(R_{h}\right) \propto p_{n}\left(R_{h}\right) \cdot V\left(R_{h}\right)$ after removing the artificial peaks in $p_{n}\left(R_{h}\right)$ by local extrapolation. Normalization sets the highest peak to 1 .

Finally, we analyze in a direct approach $g_{2}(\tau)-1$ by least-square fitting to a model of polydisperse spherical hard shell particles, undergoing independent (uncorrelated) Brownian motion, using the lsqnonlin routine of MATLAB Optimization Toolbox (Version 7.5.0.342 (R2007b), The MathWorks Inc.), dedicated to solve nonlinear least-squares problems. A bimodal size distribution was assumed, consisting of a fixed part and a freely varied Gaussian-distributed component. The constant part describes the size distribution of SVs as determined by cryo-EM, whereas the Gaussian distribution accounts for larger membranous trace particles. Again, a constant shell thickness of $12 \mathrm{~nm}$ is assumed for the particles.

Effects due to the particle concentration on the DLS data have been ruled out by dilution series on a SV preparation with an initial vesicle concentration of about $(1.98 \pm 0.03) \times 10^{14}$ vesicles $/ \mathrm{ml}$. For SV particle concentrations from $1.89 \times 10^{12}$ to $1.55 \times 10^{10}$ vesicles $/ \mathrm{ml}$ no significant effects on the autocorrelation function are visible after rescaling, see fig. 5(A) in appendix B. Further, DLS autocorrelation curves are highly reproducible for identical samples, see fig. 5(B) in appendix B.

Figure 4 shows an intensity correlation function of a native SV ensemble (black points, and error bars) and a least-squares fit to the data (solid red line). In this example, the SV dispersion was diluted to a concentration of $(2.09 \pm 0.03) \times 10^{11}$ vesicles $/ \mathrm{ml}$. The error bars correspond to the standard deviation $\sigma_{\tau}$ at each lag time $\tau$. A single exponential $y(t)=y_{0}+a e^{-t / \tau}$ (red line) was leastsquare-fitted to the data, where $y_{0}=0.0022 \pm 0.0002$, $a=0.3701 \pm 0.0004$ and $\tau=(0.616 \pm 0.003) \mathrm{ms}$. The fit yielded a reduced $\chi^{2}$ of 6.18 . From the decay time $\tau$ a particle radius of $R_{h}=104.8 \pm 0.6 \mathrm{~nm}$ was calculated according to $1 / \tau=2\left(q^{2} k_{B} T\right) /\left(6 \pi \eta R_{h}\right)$.

Although the hydrodynamic radius $R_{h}$ is approximately in the right order of magnitude, it is too large. However, it is clear from the reduced $\chi^{2}$ and the distribution of the residuals that a single exponential is not sufficient to describe the data set well. The inset in fig. 4 illustrates that there are more contributions present and that these have larger decay times. The sample appears to be polydisperse. Scattering from particles depends nonlinearly on the particle size, leading to a relative over representation of larger particles in the scattering signal. A single exponential fit will therefore be usually biased toward larger radii.

Further, a polydisperse size distribution (fig. 4(B)) was obtained from the data set shown in fig. 4(A) by a regularized nonlinear inversion of the intensity correlation function $g_{2}(\tau)-1$ modeling the particles as hollow spheres. The error bars displayed correspond to the standard deviation $\sigma_{\tau}$ at each lag time $\tau$. The inversion was performed by the ALV software, yielding first the intensity-weighted distribution $p_{I}\left(R_{h}\right)$ (black circles). Experimental errors were estimated by the ALV software according to the theoretical model described in $[32,39,40]$. Correcting for the par- 

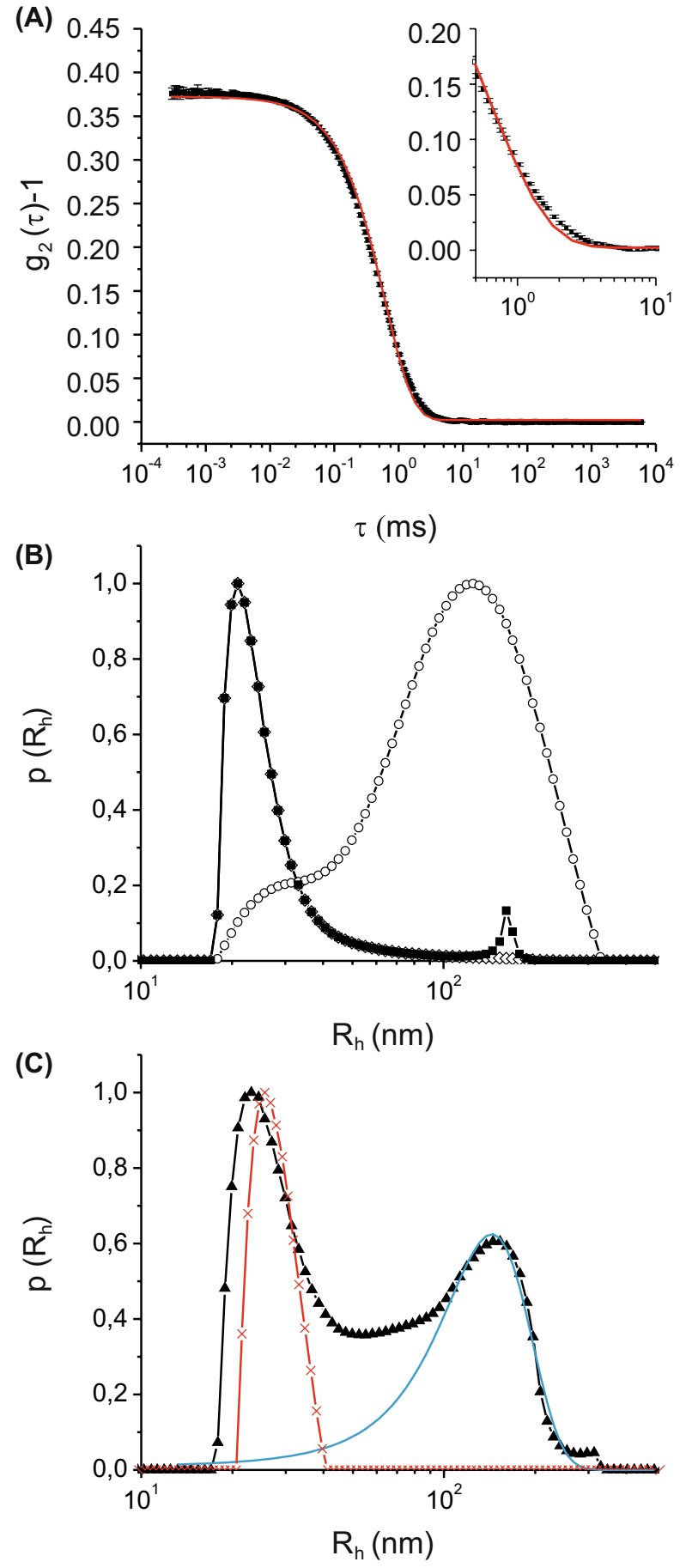

Fig. 4. (Colour on-line) (A) Intensity correlation function of a native SV ensemble (black points, with error bars) and a single exponential fit (red line). The inset shows a blow-up of part of the data. (B) Intensity-weighted distribution (black circles) and size distribution obtained by a regularized inversion of the data shown in (A) before (full black squares) and after removal of artificial peaks by local extrapolation (black empty diamonds). (C) Mass distribution (full black triangles) obtained from the size distribution (black empty diamonds) in (B) and Gaussian fit to larger particles. The mass distribution of SVs purified by AFFF fractionation (red crosses) is added to demonstrate the effect of purification. ticle volume and form factor the size distribution $p_{n}\left(R_{h}\right)$ (full black squares) was then calculated from $p_{I}\left(R_{h}\right)$.

The width and shape of the distribution $p_{n}\left(R_{h}\right)$ are to some degree influenced by the regularizor (zero order, or second order) and the regularization strength, set by the value for $P R O B 1$, see above. The maximum of $p_{n}\left(R_{h}\right)$ at $20.9 \mathrm{~nm}$ is here, however, found to be independent of the order of the regularizor and the regularization strength. A second peak at $161 \mathrm{~nm}$ arises from the correction for the particle form factor. Here the form factor takes values close to zero, making it difficult to determine the relative number of particles within that particular size range.

The mass distribution $p_{M}\left(R_{h}\right)$ of the native SV sample obtained from $p_{n}\left(R_{h}\right)$ after replacing the second artificial peak by extrapolation shows a bimodal distribution of vesicles and larger particles. The second population was fitted with a Gaussian function and yielded $45 \%$ of the total mass. For comparison a plot of SVs purified by $\mathrm{AFFF}$ fractionation ( 5 min) (see sect. 4.1) was added. Here, the contribution of larger particles to the total mass is below the resolution of the regularized inversion approach.

\section{Appendix B. Dilution series}

Figure 5(A) shows a dilution series of a sample of SVs with an original protein concentration of $3.49 \mu \mathrm{g} / \mu \mathrm{l}$, corresponding to a vesicle concentration of (1.98 \pm $0.03) \times 10^{14} \mathrm{SVs} / \mathrm{ml}$. The sample was diluted to yield the final concentrations of $(1.98 \pm 0.03) \times 10^{12} \mathrm{SVs} / \mathrm{ml}$ (black empty triangles), $(9.91 \pm 0.11) \times 10^{11} \mathrm{SVs} / \mathrm{ml}$ (red full squares), $(4.96 \pm 0.06) \times 10^{11} \mathrm{SVs} / \mathrm{ml}$ (green empty squares $),(2.48 \pm 0.03) \times 10^{11} \mathrm{SVs} / \mathrm{ml}$ (blue crosses), $(1.27 \pm$ $0.02) \times 10^{11} \mathrm{SVs} / \mathrm{ml}$ (cyan full diamonds), $(6.20 \pm 0.07) \times$ $10^{10} \mathrm{SVs} / \mathrm{ml}$ (magenta full triangles) and $(1.55 \pm 0.02) \times$ $10^{10} \mathrm{SVs} / \mathrm{ml}$ (yellow circles). The scattered intensity was recorded at a scattering angle of $90^{\circ}$ and the intensity autocorrelation functions of three consecutive intervals of $60 \mathrm{~s}$ were averaged, leading to a total measurement time of $180 \mathrm{~s}$ for each sample. For comparability the intensity correlation functions were rescaled to overlap in a lag time region around $0.02 \mathrm{~ms}$. The inset shows a selection of the correlation functions before rescaling.

The rescaled intensity correlation functions agree well and show the same decay times within the estimated experimental errors. Consequently, the dilution in our experiments has no significant influence on the calculated size distribution functions. However, the inset shows that the amplitude of the intensity correlation functions decreases with increasing dilution. The amplitude of the intensity correlation function is proportional to the average number of particles within the illuminated sample volume and is indicative of the statistical accuracy of the measurement. With increasing dilution the signal-to-noise ratio therefore decreases.

Figure 5(B) shows three DLS data sets corresponding to a sample of SVs with a protein concentration of $4.01 \times$ $10^{-4} \mu \mathrm{g} / \mu \mathrm{l}$, equivalent to $(2.28 \pm 0.03) \times 10^{10} \mathrm{SVs} / \mathrm{ml}$. For 

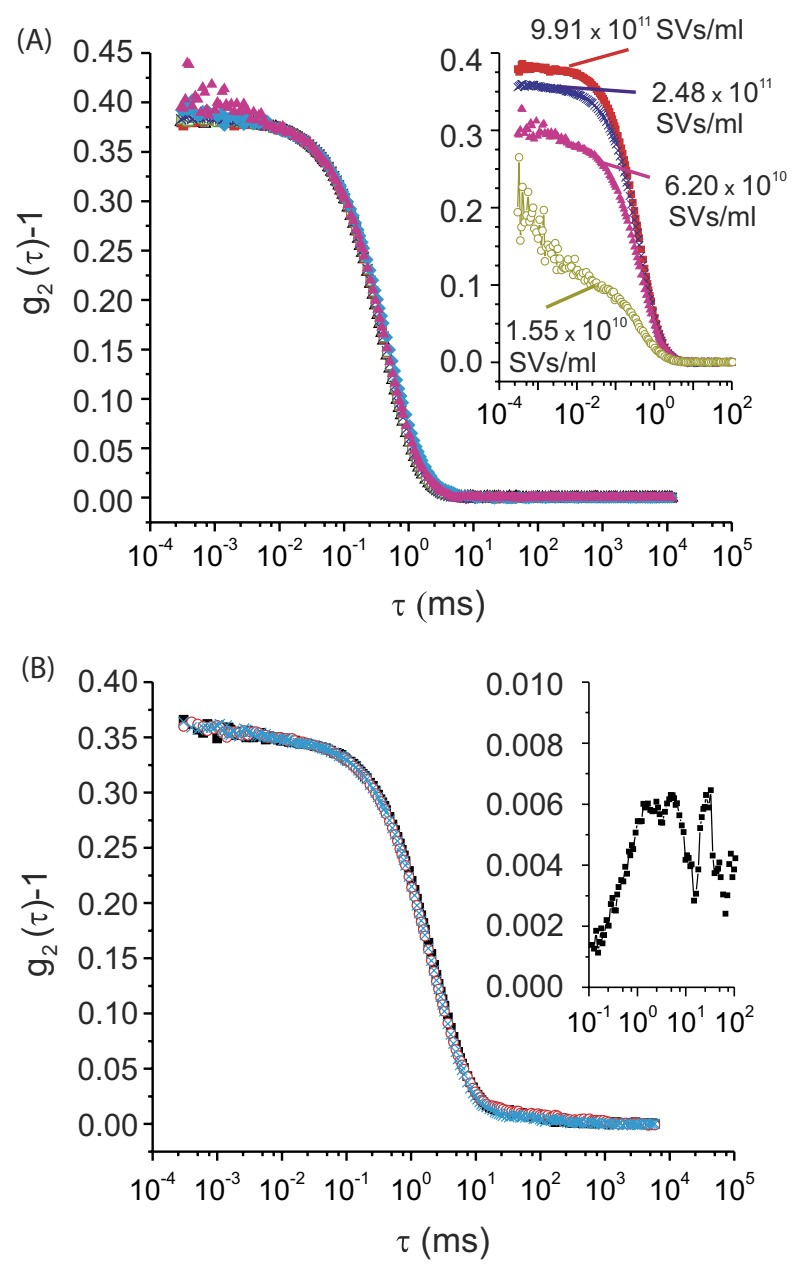

Fig. 5. (Colour on-line) (A) DLS data of a native SV ensemble at different dilution steps (black empty triangles: $(1.98 \pm 0.03) \times$ $10^{12} \mathrm{SVs} / \mathrm{ml}$, red full squares: $(9.91 \pm 0.11) \times 10^{11} \mathrm{SVs} / \mathrm{ml}$, green empty squares: $(4.96 \pm 0.06) \times 10^{11} \mathrm{SVs} / \mathrm{ml}$, blue crosses: $(2.48 \pm 0.03) \times 10^{11} \mathrm{SVs} / \mathrm{ml}$, cyan full diamonds: (1.27 \pm $0.02) \times 10^{11} \mathrm{SVs} / \mathrm{ml}$, magenta full triangles: $(6.20 \pm 0.07) \times$ $10^{10}$ vesicles/ml and yellow circles: $\left.(1.55 \pm 0.02) \times 10^{10} \mathrm{SVs} / \mathrm{ml}\right)$. The correlation functions were rescaled to overlap around $0.02 \mathrm{~ms}$. The inset shows a selection of the correlation functions before rescaling. (B) Three DLS data sets taken from native SV ensembles from the same SV preparation at a dilution of $(2.28 \pm 0.03) \times 10^{10} \mathrm{SVs} / \mathrm{ml}$. The correlation functions were rescaled to overlap around $0.1 \mathrm{~ms}$. The inset shows the maximum difference between the displayed correlation functions.

each intensity correlation function three measurements of $60 \mathrm{~s}$ were averaged, leading to a total measurement time of 180 s per function. The intensity correlation functions were rescaled to overlap in a lag time region around $0.1 \mathrm{~ms}$. The inset shows the maximum difference between the displayed correlation functions.

The three rescaled functions match each other well. Indeed, we found that this averaging over several short measurements significantly reduced the noise on the measured correlation functions. The same noise reduction is achieved by an equivalent long measurement. However, the former procedure has an advantage over the latter in that unsuited short measurements, where, e.g., a large contamination passed through the illuminated sample volume, can be easily identified and sorted out before averaging. However, care needs to be taken to chose an acquisition period for single runs that is sufficiently long that the measured correlation functions reach the baseline for a sufficiently high number of data points.

Open Access This article is distributed under the terms of the Creative Commons Attribution Noncommercial License which permits any noncommercial use, distribution, and reproduction in any medium, provided the original author(s) and source are credited.

\section{References}

1. J. Heuser, T. Reese, J. Cell Biol. 57, 315 (1973).

2. H. Lodish et al., Molecular Cell Biology (W. H. Freeman and Company, 2000).

3. B. Alberts et al., Molecular Biology of the Cell (Gerland Science, 2002).

4. T. Südhof, Annu. Rev. Neurosci. 27, 509 (2004).

5. S. Takamori et al., Cell 127, 831 (2006).

6. S. Castorph, D. Riedel, L. Arleth, M. Sztucki, R. Jahn, M. Holt, T. Salditt, Biophys. J. 98, 1200 (2010).

7. S. Castorph, L. Arleth, M. Sztucki, U. Vainio, S.K. Ghosh, M. Holt, R. Jahn, T. Salditt, J. Phys.: Conf. Ser. 247, 012015 (2010).

8. R. Jahn, T. Lang, T. Südhof, Cell 112, 519 (2003).

9. R. Jahn, R. Scheller, Nat. Rev. Mol. Cell Biol. 7, 631 (2006).

10. M. Baumert et al., J. Cell Biol. 110, 1285 (1990).

11. G. Perevucnik, P. Schurtenberger, D. Lasic, H. Hauser, Biochim. Biophys. Acta Biomembranes 821, 169 (1985).

12. K. Matsuzaki, O. Murase, K. Sugishita, S. Yoneyama, K. Akada, M. Ueha, A. Nakamura, S. Kobayashi, Biochim. Biophys. Acta Biomembranes 1467, 219 (2000).

13. D. Woodbury, E. Richardson, A. Grigg, R. Welling, B. Knudson, J. Liposome Res. 16, 57 (2006).

14. A.K. Awizio, F. Onofri, F. Benfenati, E. Bonaccurso, Biophys. J. 93, 1051 (2007).

15. E. Day, J. Ho, R. Kunze jr, S. Sun, Biochim. Biophys. Acta Biomembranes 470, 503 (1977).

16. L. Boni, J. Hah, S. Hui, P. Mukherjee, J. Ho, C. Jung, Biochim. Biophys. Acta Biomembranes 775, 409 (1984).

17. V. Trivedi, C. Yu, B. Veeramuthu, S. Francis, D. Chang, Chem. Phys. Lipids 107, 99 (2000).

18. N. Moskowitz, S. Puszkin, W. Schook, J. Neurochem. 41, 1576 (1983).

19. E. Connell, A. Giniatullina, J. Lai-Kee-Him, R. Tavare, E. Ferrari, A. Roseman, D. Cojoc, A. Brisson, B. Davletov, J. Mol. Biol. 380, 42 (2008).

20. G.L. Peterson, Anal. Biochem. 83, 346 (1977).

21. J. Giddings, Science 260, 1456 (1993).

22. M.H. Moon, J.C. Giddings, J. Pharmaceut. Biomed. Anal. 11, 911 (1993).

23. B. Korgel, J. van Zanten, H. Monbouquette, Biophys. J. 74, 3264 (1998). 
24. D.R. Arifin, A.F. Palmer, Biotechnol. Prog. 19, 1798 (2003).

25. S. Hupfeld, D. Ausbacher, M. Brandl, J. Separation Sci. 32, 1465 (2009).

26. S. Hupfeld, H.H. Moen, D. Ausbacher, H. Haas, M. Brandl, Chem. Phys. Lipids 163, 141 (2010).

27. S. Oh, D. Kang, S.-M. Ahn, R.J. Simpson, B.-H. Lee, M.H. Moon, J. Separation Sci. 30, 1082 (2007).

28. D. Kang, S. Oh, S. Ahn, B. Lee, M. Moon, J. Proteome Res. 7, 3475 (2008).

29. M. Holt, D. Riedel, A. Stein, C. Schuette, R. Jahn, Curr. Biol. 18, 715 (2008).

30. L. Mandel, Fluctuations of Light Beams, Vol. II (NorthHolland Publishing Company, 1963).

31. B. Berne, R. Pecora, Dynamic Light Scattering (Dover Publications Inc., 2000).
32. W. Brown, Dynamic Light Scattering: The Method and Some Applications (Oxford University Press, USA, 1993).

33. G. van den Bogaart, M. Holt, G. Bunt, D. Riedel, F. Wouters, R. Jahn, Nat. Struct. Mol. Biol. 17, 358 (2010).

34. C. Baker, L. Fox, D. Mayers, K. Wright, Comput. J. 7, 141 (1964).

35. J. McWhirter, E. Pike, J. Phys. A: Math. Gen. 11, 1729 (1978).

36. J. Honerkamp, J. Weese, Continuum Mech. Thermodyn. 2, 17 (1990).

37. S. Provencher, Comput. Phys. Commun. 27, 213 (1982).

38. S. Provencher, Comput. Phys. Commun. 27, 229 (1982).

39. K. Schatzel, M. Drewel, S. Stimac, J. Mod. Opt. 35, 711 (1988).

40. K. Schatzel, Quantum Opt.: J. Eur. Opt. Soc. B 2, 287 (1990). 